
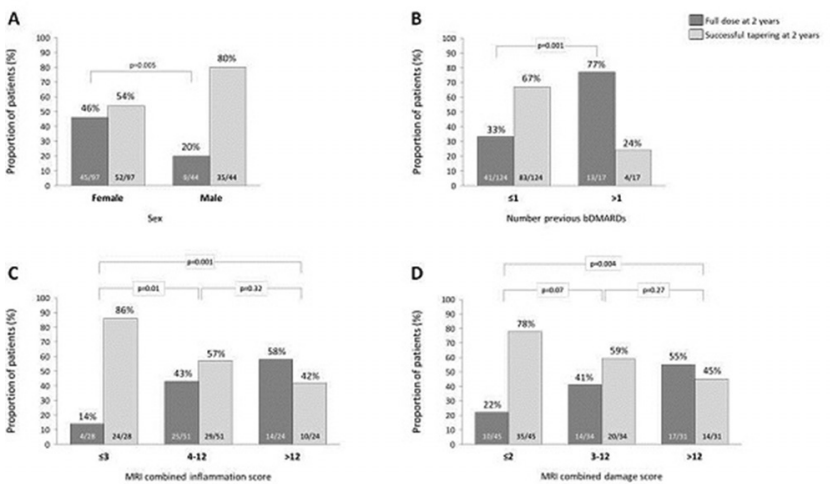

Abstract OP0038 - Figure 1 Observed association between potential predictors and the proportion patients eith successful tapering (i.e. less than full dose) versus full dose at year 2

Methods: One-hundred-and-forty-three patients with sustained disease activity score (DAS28-CRP) $\leq 2.6$ and no radiographic progression the previous year were included. bDMARD was reduced to $2 / 3$ of standard dose at baseline, $1 / 2$ after 16 weeks, and discontinued after 32 weeks. Patients who flared (defined as either $D A S 28-C R P \geq 2.6$ and DAS28-CRP $\geq 1.2$ from baseline, or erosive progression on $\mathrm{X}$-ray and/or MRI) stopped tapering and were escalated to the previous dose level.

Results: One-hundred-and-forty-one patients completed 2 year follow-up. At 2 years, 87 patients (62\%) had successfully tapered bDMARDs, with $26(18 \%)$ receiving $2 / 3$ of standard dose, $39(28 \%) 1 / 2$ dose and $22(16 \%)$ having discontinued; 54 patients $(38 \%)$ were receiving full dose. DAS28-CRP ${ }_{0-2 y r s}$ was 0.1 $((-0.2)-0.4)$ (median(interquartile range)) and mean Total-Sharp-Score ${ }_{0-2 y r s}$ was 0.01 (1.15) (mean(SD)). Radiographic progression was observed in 9 patients (7\%). Successful tapering was independently predicted by: $\leq 1$ previous bDMARD, male gender, low baseline MRI combined inflammation score and low $\mathrm{MRI}$ combined damage score. Negative IgM-rheumatoid factor predicted successful discontinuation. The association between potential predictors and the proportion of patients with successful tapering of bDMARDs is shown in figure 1 . Conclusions: By implementing a clinical guideline, $62 \%$ of RA patients in sustained remission in routine care were successfully tapered, including $16 \%$ successfully discontinued at 2 years. Radiographic progression was rare. IgM-RF was an independent predictor for successful discontinuation of bDMARDs. Maximum one bDMARDs, male gender, and low baseline MRI combined inflammation and MRI combined damage scores were independent predictors for successful tapering.

Disclosure of Interest: None declared

DOI: 10.1136/annrheumdis-2018-eular.2901

\section{OP0039 HIGH DISEASE ACTIVITY AND DISABILITY AT ONE YEAR IN TWO CLUSTERS OF PATIENTS WITH RHEUMATOID ARTHRITIS DEFINING THEMSELVES AS IN AN ACCEPTABLE STATE AT TREATMENT INITIATION}

J. Gwinnutt ${ }^{1}$, K. Hyrich ${ }^{1,2}$, M. Lunt ${ }^{1}$, A. Barton ${ }^{2,3}$, S. Verstappen ${ }^{1,2}$, on behalf of RAMS coinvestigators. ${ }^{1}$ Arthritis Research UK Centre for Epidemiology, The University of Manchester, ${ }^{2}$ NIHR Manchester Biomedical Research Centre, Manchester University Hospitals NHS Foundation Trust, ${ }^{3}$ Arthritis Research UK Centre for Genetics and Genomics, The University of Manchester, Manchester, UK

Background: A significant proportion of patients with rheumatoid arthritis (RA) define themselves as in a 'patient acceptable symptom state' (PASS) at methotrexate (MTX) initiation. Within this heterogeneous group, there are likely to be clinical phenotypes associated with poor outcome.

Objectives: To identify distinct phenotypes of symptoms within patients in PASS at baseline and to compare disability and disease activity scores of these patients over one year.

Methods: The Rheumatoid Arthritis Medication Study (RAMS) is a one year prospective cohort of patients with RA starting MTX. At baseline, patients reported demographics, completed the Health Assessment Questionnaire (HAQ), pain/ fatigue visual analogue scales (VAS) and the Hospital Anxiety and Depression Scale (HADS). A research nurse conducted a 28 swollen and tender joint count and the Disease Activity Score (DAS28) was calculated. DAS28 and HAQ were assessed again at 12 months. Patients also answered the dichotomous question 'Is your current condition satisfactory, when you take your general functioning and your current pain into consideration?' Only those answering yes at baseline were included in the analysis. Phenotypes were identified using K-medians cluster analysis based on baseline swollen/tender joint count, HAQ, VAS-pain, VASfatigue and HADS-depression. The 'elbow method' was used to select the number of clusters. Quantile regression was used to compare the 12 month HAQ and DAS28 scores between clusters, controlling for age and gender.

Results: Five clusters were identified within the 300 patients in PASS at baseline (mean (sd) age=61.4 (12.1) years, 186 (62\%) women) (table 1). Compared to Cluster 1, patients in higher clusters had worse HAQ (median difference $(95 \% \mathrm{Cl}$ ) vs Cluster 1: Cluster $2=0.36(0.11,0.61)$; Cluster $3=0.19(-0.11,0.49)$; Cluster $4=0.74(0.47,1.00)$; Cluster $5=0.89(0.54,1.24))$ and worse DAS28 at 12 months (median difference $(95 \% \mathrm{Cl})$ vs Cluster 1 : Cluster $2=0.43(-0.06,0.91)$; Cluster $3=0.40(-0.19,0.99)$; Cluster $4=0.89(0.36,1.41)$; Cluster $5=1.28(0.59,1.96)$.

Abstract OP0039 - Table 1 Baseline characteristics of the five clusters

\begin{tabular}{|c|c|c|c|c|c|c|c|c|c|c|c|c|c|}
\hline Cluster & $\mathrm{N}$ & SJC28 & $\begin{array}{r}B 2 \\
T \mathrm{CO} 8\end{array}$ & line sco & 5, Media & Fatigue & Depr & S5C28 & & ical cha & ieristic & atrix $\$$ & Depr \\
\hline 1 & 50 & 2 & 1 & 0 & 8 & 8 & 0 & M & I & $\mathrm{L}$ & L & I & L \\
\hline 2 & 109 & 2 & 3 & 0.5 & 25 & 25 & 4 & M & M & M & $M$ & M & $M$ \\
\hline 3 & 44 & 13.5 & 10.5 & 0.5 & 20 & 12.5 & 2 & H & H & M & $M$ & $M$ & $M$ \\
\hline 4 & 71 & 4 & 5 & 1.13 & 50 & 64 & 7 & M & M & H & H & H & H \\
\hline 5 & 26 & 12 & 23 & 1.25 & 56.5 & 66 & 5 & H & $\mathrm{H}$ & H & $\mathrm{H}$ & H & $M$ \\
\hline
\end{tabular}

Conclusions: Despite all patients reporting they were satisfied with their condition at baseline, five distinct clinical phenotypes were identified. These clusters can identify 'reticent' patients who are likely to have poor outcomes in the future. Disclosure of Interest: None declared DOI: 10.1136/annrheumdis-2018-eular.5164

\section{OP0040 SYNOVIAL CELL INFILTRATION IN ACPA-VE PATIENTS DISPLAYS SIMILAR SIGNATURES TO OTHER SERONEGATIVE INFLAMMATORY ARTHRITIS. RESULTS FROM THE PATHOBIOLOGY OF EARLY ARTHRITIS COHORT (PEAC)}

G. Lliso-Ribera ${ }^{1}$, F. Humby ${ }^{1}$, A. Nerviani ${ }^{1}$, M.A. Boutet ${ }^{1}$, S. Kelly ${ }^{2}$, M. Bombardieri ${ }^{1}$, M. Lewis ${ }^{1}$, R. Hands ${ }^{1}$, V. Rocher ${ }^{1}$, F. Bene ${ }^{1}$, C. Buckley ${ }^{3}$, P.C. Taylor ${ }^{4}$, I. B. Mclnnes ${ }^{5}$, C. Pitzalis ${ }^{6} .{ }^{1}$ Experimental Medicine and Rheumatology, William Harvery Research Institute. Queen Mary University London; ${ }^{2}$ Rheumatology Department, Barts Health NHS Trust, London; ${ }^{3}$ Division of Immunity and Infection, University of Birmingham, Birmingham; ${ }^{4}$ Kennedy Institute of Rheumatology, University of Oxford Botnar Research Centre, Oxford; ${ }^{5}$ Glasgow Biomedical Research Centre, University of Glasgow, Glasgow; ${ }^{6}$ Experimental Medicine and Rheumatology, William Harvery Research Institue. Queen Mary University London, London, UK

Background: There is increasing evidence to suggest that ACPA +ve and ACPA-ve RA are distinct diseases. Current data demonstrates overlap in classification criteria between ACPA-ve RA and other sero negative inflammatory arthritidies such as PsA. Associated with this is a variable prognosis and response to treatment for patients with ACPA-ve RA. Biomarkers capable of refining diagnosis and improving on current classification criteria early in the disease course for patients with ACPA-ve RA are thus urgently needed. Data examining the synovia pathophysiological relationship between PSA and ACPA $\pm R A$ is currently limited although has the potential to identify disease specific synovial cellular and molecular signatures.

Objectives: Therefore, the aim of this study is to examine in a cohort of therapy naïve, early inflammatory arthritis patients, whether ACPA-ve RA can be defined at disease initiation according to synovial pathobiological signatures.

Methods: A total of 186 consecutive DMARD naïve inflammatory arthritis patients (disease duration $<1$ year) recruited as part of the multicentre PEAC study at Barts Health NHS Trust were evaluated. All patients underwent a baseline synovial biopsy of a clinically active joint along with collection of inflammatory markers (CRP). Following $\mathrm{H}$ and $\mathrm{E}$ staining, sections underwent immumohistochemical staining and semi-quantitative scoring $(0-4)$ to determine the degree of CD20 +Bcells, CD3 + T cells, CD68 + lining (I) and sublining (sl) macrophage and CD138 +plasma cell infiltration. Sections were categorised into three pathotypes: (i) Fibroid(F):(CD68 SL $<2$ and or CD3, CD20, CD138 <1), (ii) Myeloid(M): (CD68SL $>2$, CD20 <1 and or CD3 >1) and (iii) Lymphoid(L):(grade 23 CD20 +aggregates, CD20 >2)

Results: 90/186 patients were classified as ACPA+ve RA, 55/186 as ACPA-ve RA and $41 / 186$ as PsA. $80 \%$ of synovial samples were collected from small joints (wrist, MCP, PIP). All 186 samples were suitable for analysis. Results confirmed that $\mathrm{C}$-reactive protein (CRP) as inflammatory marker does not differentiate between subgroups ( $p$ 0.41). Significantly higher degree of immune cell infiltration was seen between ACPA+ve vs ACPA-ve and ACPA+ve vs PsA but not between ACPA-ve and PsA (figure 1). When grouping patient between clinical subgroups 